\title{
Traduire
}

Revue française de la traduction

$230 \mid 2014$

À la croisée du texte et de l'image

\section{Traduire les albums pour enfants : Relation texte- image dans les traductions italienne et française de In the Night Kitchen}

\section{Margherita Ippolito}

Traducteur : Hélène Ladjadj

\section{OpenEdition Journals}

\section{Édition électronique}

URL : http://journals.openedition.org/traduire/627

DOI : $10.4000 /$ traduire. 627

ISSN : 2272-9992

Éditeur

Société française des traducteurs

Édition imprimée

Date de publication : 15 juin 2014

Pagination : 75-86

ISSN : 0395-773X

Référence électronique

Margherita Ippolito, «Traduire les albums pour enfants : Relation texte-image dans les traductions italienne et française de In the Night Kitchen », Traduire [En ligne], 230 | 2014, mis en ligne le 15 juin 2016, consulté le 19 avril 2019. URL : http://journals.openedition.org/traduire/627 ; DOI : 10.4000/ traduire.627 


\section{Traduire les albums pour enfants : Relation texte-image dans les traductions italienne et française de In the Night Kitchen}

\section{Margherita Ippolito}

À vrai dire, un livre illustré, c'est un poème visuel.

Maurice Sendak

Le présent article(1) s'intéresse aux conséquences potentielles du processus de traduction sur les relations complexes qu'entretiennent les mots et les images. II constate que la traduction des albums est une forme de traduction particulière qui dépend du statut sémiotique des signifiants visuels et verbaux dans le texte. Les traducteurs doivent se préoccuper, non seulement du message verbal, mais aussi de l'interaction texte-image pour reconstruire la dynamique interne d'un album dans la langue cible, s'ils veulent respecter les intentions de l'auteur et donner une vie nouvelle à la complexité de l'œuvre originale. Si le traducteur peut produire une série de glissements lexico-sémantiques pour le texte, les images, elles, ne peuvent être soumises à aucune transposition, ni linguistique ni culturelle. Pour cette étude, nous avons analysé les traductions italienne et française de In the Night Kitchen (1970), de Maurice Sendak, afin de mettre en évidence tout ce qu'impliquent les liens entre les mots et les images pour l'opération de traduction.

\section{Association visuel-verbal}

Selon Maurice Sendak, In the Night Kitchen est né de l'un de ses grands rêves d'enfant : observer les boulangers qui travaillent la nuit.

Lorsque j'étais enfant, il y avait une réclame qui disait : "Nous faisons le pain pendant que vous dormez! " Cela me semblait particulièrement sadique car je n'avais qu'une envie, ne pas dormir pour pouvoir regarder. [...] J'étais vraiment très contrarié et je me souviens que je gardais les coupons montrant les trois petits boulangers dodus de la marque Sunshine en route, la nuit venue, vers ce lieu magique, situé Dieu sait où, pour s'amuser tandis que je devais aller au lit. Ce livre est une sorte de revanche par lequel je leur fais savoir que maintenant je suis

(1) La version originale anglaise de cet article est parue dans la revue Norwich Papers, vol. 21 (2013). 
assez grand pour veiller et donc découvrir ce qui se passe dans la cuisine la nuit. (Sendak in Lanes, 1980, p. 174)

Dans ce livre, l'histoire révèle "pourquoi [...] on ne peut pas avoir de gâteaux le matin ", comme l'explique le narrateur à la dernière page. L'histoire a néanmoins un sens beaucoup plus profond, lié à l'identification et à la confusion des jeunes enfants face à la nourriture, un élément fondamental des premières étapes du développement concernant l'acquisition progressive d'une identité autonome et distincte. In the Night Kitchen est un conte onirique présenté sous la forme d'un rêve fait par le jeune héros, Mickey. C'est le travail inconscient qui s'opère pour qu'un enfant acquière le sens de son individualité, passant de la phase orale, représentée par la fixation sur la nourriture, à une expression plus évoluée du soi. Quand l'histoire commence, c'est la nuit. Mickey dort et soudain, il est réveillé par un grand bruit. Après avoir perdu son pyjama, il tombe dans le grand pétrin contenant la pâte, dans la cuisine, la nuit. Trois sosies boulangers le prennent pour du lait et le mélangent à la pâte en chantant : Milk in the batter! Milk in the batter! [Du lait dans mon pétrin ! Du lait dans mon pétrin !] Puis, Mickey se retrouve au four, prêt à cuire, mais parvient à se libérer en revendiquant son identité : I'm not the milk and milk's not me! l'm Mickey! [Je ne suis pas du lait, rien à voir avec moi ! Moi, je suis Mickey !] Mais les boulangers tiennent à leur lait et s'approchent de Mickey, l'air menaçant. Heureusement, l'enfant s'envole dans l'avion qu'il a fabriqué avec la pâte, en criant : I'm Mickey the pilot! I get milk the Mickey way [Je suis Mickey le pilote ! Le lait, j'en trouve façon Mickey, sur la voie lactée(2).] II s'empare de la mesure des boulangers et survole la voie lactée

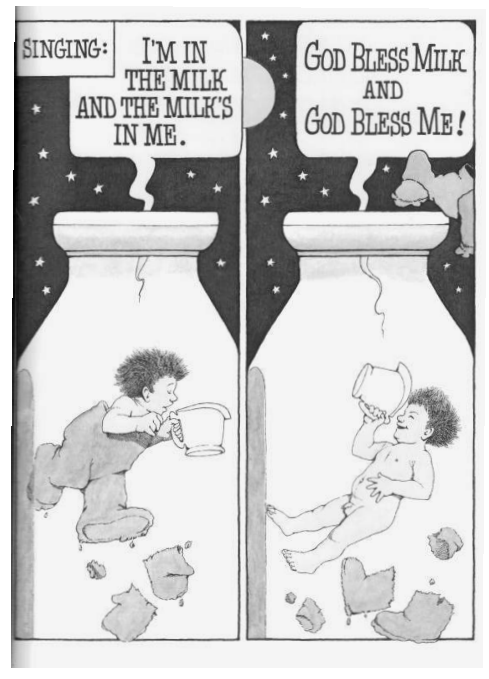

(2) En même temps qu'une allitération, il y a ici (et tout au long du texte) un jeu de mots en anglais sur le nom du jeune garçon (Mickey) et la voie lactée (Milky way) : la voie lactée devient la voie de Mickey! 
de la cuisine de nuit - une bouteille de lait géante - dans laquelle il plonge et se met à nager joyeusement. Pour finir, il verse du lait de la bouteille géante dans la pâte et les boulangers peuvent alors pétrir le gâteau.

Dans ce livre, les images dominent, occupant l'essentiel de la page. Le texte est présent sous diverses formes : bulles contenant les propos tenus par les personnages, légendes réservées aux interventions du narrateur, paratexte scripto-visuel incluant les représentations onomatopéiques des sons et les inscriptions, ces dernières constituant la forme la plus problématique. Ainsi, sur l'une des doubles pages, les seuls mots présents le sont sur les étiquettes et inscriptions des bouteilles, cartons et boîtes dans la cuisine. En général, la partie picturale s'impose par de grandes illustrations très colorées. Mais l'histoire de Sendak ne saurait être racontée par les dessins ni par les mots seuls, car elle progresse par une étroite interaction entre les deux éléments qui sont indissociables pour la compréhension. L'intégration des codes verbaux et visuels constituent donc le "concept total " (Mœbius 1990, p. 133), une œuvre intégrant deux codes qui se modifient l'un l'autre : "La relation texte-image ne relève pas tant d'une question d'équilibre que de la façon dont texte et image interagissent et se transforment réciproquement. " (Sipe 1998, p. 98).

Le lecteur décode simultanément les signes verbaux et visuels pour établir le lien fondamental entre eux. Page après page, ses yeux passent d'une séquence d'images et de mots interdépendants à l'autre, explorant un réseau de significations dont l'union donne son unité à la narration. L'expérience de lecture devient alors une activité captivante et créative, le lecteur ayant un rôle actif dans l'ordonnancement de la relation mot-image. L'album s'inscrit dans une zone de contact aux dimensions multimodales, à partir des messages transmis simultanément par les mots et les illustrations. C'est le lecteur qui établit ce contact entre le verbal et le visuel et qui perçoit les significations implicites. Cette zone de contact est également conçue comme un espace, comme l'explique Sendak à propos de sa façon de créer des albums :

II ne faut jamais représenter exactement ce que dit le texte. II faut laisser de l'espace dans le texte pour que les images puissent jouer leur rôle. Alors, vous donnerez la priorité aux mots lorsqu'ils sont plus efficaces. En fait, c'est une façon de jongler qui exige une grande technique et beaucoup d'expérience pour garder le tempo. (Sendak in Lanes 1990, p. 110)

On ouvre ainsi une gamme infinie de possibilité de lectures en " jonglant " avec le changement constant des fonctions de connotation et dénotation du langage : de nouveaux détails apparaissent à chaque lecture de l'image par le lecteur ; les illustrations suggèrent d'autres significations et, surtout, la relation entre texte et illustrations oriente vers d'autres interprétations, la plupart du temps multiples. La tension entre les représentations diégétique et mimétique enrichit l'interaction entre les deux médias avec des possibilités de communication inédites et inattendues : "Un album invite à maintes lectures et ainsi, au lieu de ne livrer qu'une histoire, il permet d'en inventer plusieurs" (Meek 1992, p. 33). 


\section{Traduire l'interaction des messages visuels et verbaux}

In the Night Kitchen appartient à la catégorie des " récits interdépendants " de Denise Agosto dans lesquels visuel et verbal ne peuvent être considérés comme deux entités distinctes, contrairement aux "récits parallèles ", mais constituent un système sémiotique complexe et indissociable (Agosto 1999, p. 267). Dans le domaine du récit interdépendant, Agosto distingue deux dynamiques : "l'augmentation et la contradiction " (Agosto 1999, p. 269-270). La première forme la structure d'In the Night Kitchen, les images enrichissant et décuplant le sens exprimé par les mots et réciproquement, les mots amplifiant et élargissant ce que montrent les images, tandis que la seconde, avec laquelle les mots et les images apportent des informations contradictoires, en est absente. Cependant, l'augmentation est une dynamique très complexe qui exige des capacités d'interprétation particulières : la lecture des éléments verbaux seuls est inutile et dénuée de sens car l'histoire ne serait pas compréhensible. C'est pourquoi le traducteur ne peut se limiter aux mots mais doit prendre en compte les relations entre mots et images. Autrement dit, le traducteur, outre ses compétences linguistiques et culturelles, doit aussi maîtriser la langue des images et les interactions possibles entre les codes verbaux et visuels.

La traduction requiert donc une approche multimodale qui suppose :

1) une analyse précise des différents moyens employés pour transmettre le sens ;

2) l'étude des interactions entre les modes de narration, sachant que "les modes sont des ressources sémiotiques qui permettent la présentation simultanée de discours et de types d'(inter)action " (Kress, van Leeuwen 2001, p. 21) ;

3) le transfert, dans le sens de déplacement, des interactions entre les divers modes dans le texte cible pour obtenir un effet identique (ou au moins similaire) à celui du texte source : "Le lecteur de la traduction idéale doit avoir à faire le même travail que le lecteur de la version originale " (O'Sullivan 2006, p. 113). Plus le travail du lecteur du texte source est complexe, - autrement dit lorsque les dynamiques verbal-visuel s'accumulent et offrent des interprétations multiples - plus la tâche du traducteur est difficile. Riitta Oittinen souligne que : " La traduction des textes d'albums est un domaine spécifique, sinon une langue particulière, qui exige une spécialisation, associée à une formation en art par exemple " (Oittinen 2000, p. 114).

L'essentiel du sens dans In the Night Kitchen provient du glissement du rôle des signifiants et de leurs signifiés, un jeu centré autour de l'association Mickey/milk. Ces deux entités sont souvent confondues ou interverties. D'abord, le prénom Mickey et le nom commun milk forment un jeu de mots hétérophonique, les deux mots ayant une consonance différente et cependant assez proche du fait de la répétition des consonnes et du son de la voyelle (/m/, /k/, /i/). Ensuite, ce jeu de mots évolue et prend différentes formes, l'un suggérant ou signifiant l'autre, une inversion exprimée sur le plan verbal comme sur le plan visuel : les boulangers prennent Mickey pour du lait parce que l'enfant semble aussi blanc que du lait, et par ses actes Mickey est représenté comme formant un tout avec le lait. 
Les images représentent clairement cette association (sur une page Mickey plonge et s'amuse dans une grande bouteille de lait ; sur la couverture, il est représenté avec une mesure de lait sur la tête et, à la dernière page, on le voit entourant une bouteille de lait de ses bras). Sur le plan verbal, Mickey chante : I'm in the milk and the milk's in me. Le jeu de mot Mickey-milk génère d'autre jeux de mots comme Mickey-cake et milk cake, Mickey way et Milky Way, ainsi que des rimes et des chansons, comme "I'm in the milk and the milk's in me. God Bless Milk and God Bless me!" [Je suis dans le lait et le lait est en moi. Dieu bénisse le lait et Dieu me bénisse !] dans lesquelles l'identification lait-moi est manifeste, et le refrain du boulanger "Milk in the batter! Milk in the batter! We bake cake! And nothing's the matter!" [Du lait dans le pétrin! Du lait dans le pétrin ! Nous faisons un gâteau ! Et tout va bien !] où nothing devient le sujet ou l'objet (matter) de l'énonciation tandis que le processus d'identification/confusion atteint sa conclusion inévitable.

Fait significatif, ce refrain est chanté par les pâtissiers qui confondent maintenant les ustensiles de cuisine avec des instruments de musique, tout comme ils ont confondu Mickey avec du lait. La connexion entre milk et Mickey, au-delà du jeu de mots et de la fusion visuelle des signifiants, déclenche une série d'associations d'idées en termes de connotation et de dénotation : le lait évoque le premier aliment des petits, le début de l'existence, le symbole de l'attention, de la protection et de l'amour maternel. Mickey nageant dans le lait renvoie à la fois à l'immersion dans le liquide amniotique et au baptême qui préside à une nouvelle vie. Le lait représente aussi le petit-déjeuner, avec la bonne odeur du lait, des biscuits, de la confiture, du gâteau et du sucre parfumant l'atmosphère.

\section{La traduction en italien}

La première occurrence de fusion Mickey-lait est un moment clé dans l'histoire : "the bakers who bake till dawn so we can have cake in the morn mixed Mickey in batter chanting: Milk in the batter! Milk in the batter!" Sur les images, Mickey est déjà dans la pâte et la confusion par les trois boulangers de Mickey avec le lait n'est expliquée ni dans le texte ni sur l'image. L'événement se suffit à lui-même.

La traduction italienne s'éloigne systématiquement de l'original : " / cuochi, vedendo cascare quella cosa bianca nella pasta, cominciarono subito a mescolare cantando: Latte nella focaccia! Latte nella focaccia! " [Les cuistots, voyant cette chose blanche tomber dans la pâte commencent alors à mixer en chantant: Du lait dans la focaccia! Du lait dans la focaccia!]. Les stratégies de traduction utilisées - amplification, omission et localisation - tendent à éliminer la tension mot-image. La traductrice(3) opère des glissements qui orientent le lecteur vers une

(3) Selene Maltini a traduit le texte en italien. 
interprétation unique et non multiple, explicite plutôt qu'ambiguë, du texte, avec une intervention expliquant que les trois boulangers confondent le jeune garçon avec du lait tout simplement parce qu'il est blanc. Ce choix, qui révèle le point de vue de la traductrice et son interprétation du texte, n'était pourtant pas nécessaire car le texte source n'exige pas cette explication. Cette intrusion de la traductrice simplifie le texte à l'excès car elle réduit les possibilités d'interprétation du lien entre l'enfant et le lait à la plus évidente et la plus simple : la couleur. Le lecteur ne pourra saisir et apprécier le réseau de signes semés dans la narration car les défis visuel ou verbaux et les aspects inattendus ou absurdes liés au protagoniste (et au lait), qui forment la structure profonde du texte, ont disparu.

Le choix de la localisation nuit aussi à l'interaction verbal-visuel. La traductrice préfère remplacer le prénom Mickey par le prénom italien Luca, qui offre peu de lien avec latte [lait]. Du point de vue phonétique le lien entre le prénom de l'enfant et le lait n'existe plus, l'allitération de la consonne initiale /// ne suffisant pas à créer un écho phonétique entre les deux mots. La localisation va bien au-delà de ce choix onomastique puisqu'elle intervient aussi sur ce que préparent les boulangers, le morning cake devenant un aliment typiquement italien, la focaccia. Ce choix entraîne plusieurs problèmes quant au sens : le lait n'est pas un ingrédient de la focaccia, sorte de galette souple faite de farine, sel, levure, huile et éventuellement parsemée de tomates, d'olives ou d'herbes. Tout au plus peut-on ajouter un peu de lait pour rendre la pâte plus souple. En outre, sur le plan visuel, une grosse boule de pâte brune est découpée en petites boules rondes que les boulangers mettent au four et qui n'ont rien de commun avec la forme de la focaccia, plate et blanche. Cette contradiction entre le texte et les images multiplie les incohérences déroutantes pour le lecteur.

Ces choix de traduction obligent alors la traductrice à s'éloigner du texte à plusieurs reprises. En effet, la focaccia n'est pas consommée au petit-déjeuner ; la traductrice doit donc supprimer toute référence au petit-déjeuner - c'est pourquoi elle a supprimé who bake till dawn so we can have cake in the morn. II lui faudra même modifier la fin de l'histoire. Sur la dernière page de la version originale, le narrateur souligne que cette histoire explique pourquoi on ne peut pas avoir de gâteau le matin, ce qui, dans le texte cible, devient : "[Luca] già sognava la sua grande avventura di latte, di luna e di focaccia" [(Luca) rêvait déjà de sa grande aventure avec le lait, la lune et la focaccia]. La traduction n'a plus rien de commun avec la version originale et illustre combien l'approche choisie oriente tout le processus de traduction qui devient une forme de re-création, une réécriture autonome de (certaines parties) du texte source. Les illustrations demeurent, mais l'histoire devient autre chose. Le code verbal est traité indépendamment du code visuel, faisant de l'album un livre illustré, les images devenant une simple répétition ou ornementation du texte.

La référence à la lune n'est pas réservée à la fin de l'histoire. La lune devient en effet un élément important dans le texte cible. Elle est présente dès le titre, Luca, la luna e il latte [Luca, la lune et le lait], comme dans plusieurs séquences narratives et discours indirects. Ainsi, au 
début du texte source, le narrateur raconte comment Mickey "fell through the dark, out of his clothes past the moon and his mama and papa sleeping tight", tandis que la traductrice précise que Luca "improvvisamente scivolò nelle tenebre, fuori dal suo pigiama e bianco come il latte nella luce della luna " [tombe soudain dans le noir sans son pyjama, et il est aussi blanc que du lait au clair de lune]. Outre la suppression des parents du protagoniste, si importants pour le fondement mythique du texte original, la traductrice ajoute encore son interprétation personnelle et établit un lien entre Luca, milk et the moon qui détermine tous ses choix de traduction ultérieurs. De plus, la lune réapparaît lors d'un épisode plutôt dramatique lorsque l'enfant, dans la bouteille de lait, exprime en chantant son désir de bénédiction - lien avec le baptême - en remerciant Dieu pour la nourriture et l'autonomie : "I' $m$ in the milk and the milk's in me, God Bless Milk and God Bless me!" Dans le texte cible, cela devient une chanson monotone et dénuée de sens : "Latte di Luca luna di latte! Luca di latte latte di luna!" [Lait de Luca lune de lait! Luca de lait lait de lune !] reposant sur la seule répétition d'éléments lexicaux.

Le choix de la traductrice peut être compris comme une tentative de reproduire la fusion Mickey-milk par l'utilisation simultanée de la proximité verbale et de la répétition. Cependant, dans le texte original, la connexion entre ces deux éléments lexicaux n'est pas simplement phonétique mais hautement symbolique, suggérant d'autres niveaux multiples de signification. La relation Luca, luna et latte ne conserve pas la dimension symbolique de l'original car le seul point commun entre Luca et la lune, la lune et le lait et enfin Luca et le lait est, encore une fois, la couleur : en italien le blanc est en effet associé à la lune - on dit bianco come la luna [blanc comme la lune] - et au lait, bianco come il latte [blanc comme du lait].

\section{La traduction en français}

La traduction française(4) s'écarte également du texte source, même si, au moins dans la scène fondamentale où s'établit le lien entre le lait et Mickey, elle respecte la combinaison des dimensions verbale et visuelle :

The bakers who bake till dawn so we can have cake in the morn mixed Mickey in batter chanting: Milk in the batter! Milk in the batter! Stir it! Scrape it! Make it! Bake it! [Les boulangers travaillant jusqu'à l'aube pour que nous ayons du gâteau le matin mélangent Mickey à la pâte en chantant : Du lait dans la pâte ! Du lait dans la pâte ! Bien remuer ! Bien racler ! Bien dorer !]

Dans le pétrin de la cuisine

trois pâtissiers de bonne mine

mêlent les œufs et la farine,

et chantent:

Pour faire de bons pains au lait,

battez bien fort, faites lever...

(4) Jean-Henri Potier a traduit le texte en français. 
En terme de dissemblances entre les textes source et cible, le traducteur a tendance à verbaliser ce que montre l'image, s'écartant donc nettement du choix de Sendak d'une zone de contact implicite entre image et texte. Le pétrin et les trois boulangers qui deviennent " pâtissiers " parce qu'ils en portent la coiffe, sont décrits par le texte : leur aspect est explicité dans la version française du texte. Ensuite, le traducteur compose un court poème en trois octosyllabes ("Dans le pétrin de la cuisine/ trois pâtissiers de bonne mine/ mêlent les œufs et la farine "), créant un effet de rimes qui n'existe pas dans le texte source. Enfin, et surtout, si aucune stratégie de naturalisation n'est appliquée au prénom Mickey (l'enfant reste Mickey dans le texte français), il n'est plus lié au lait mais considéré comme un simple ingrédient du pain au lait. La fusion Mickey-lait qui ébranle le couple traditionnel signifiant-signifié (est-ce le lait qui signifie Mickey ou Mickey qui signifie le lait ?) disparaît complètement puisque le français n'offre aucun écho, ni phonétique ni lexical, entre Mickey et lait. Plus loin, lorsque l'enfant affirme son identité dans la chanson commentée ci-dessus, il dit : " Je ne suis pas du sucre, ni du lait, ni du beurre. Je suis Mickey. N'ayez pas peur. " La dernière phrase "N'ayez pas peur ", semble totalement superflue dans la narration, tant sur le plan linguistique qu'iconographique. Si le thème principal de l'identification entre l'enfance et la nourriture est conservé, il n'est pas rendu explicite. II est seulement suggéré par la relation entre les codes verbal et visuel. Dans cette traduction, le lecteur du texte cible doit interpréter le lien mot-image comme le fait le lecteur du texte source, afin de trouver les voies possibles dans le déroulement de la narration. II doit ainsi rassembler les divers indices verbaux et visuels pour comprendre qu'au début, l'enfant est "matière ", substance physique dépourvue d'identité, et que tous ses efforts vont donc se concentrer sur son développement émotionnel afin d'échapper à la tentative assez menaçante des boulangers d'anéantir l'identité de l'enfant dans la pâte avec leur cri inquiétant nothing's the matter! [Ça n'est rien].

Malheureusement, le fait que dans la traduction française, Mickey ne soit pas lié au lait, mais simplement l'un des ingrédients du pain au lait, entraîne quelques incohérences. De ce fait, certaines séquences narratives semblent absurdes. Par exemple, pourquoi les trois boulangers attendent-ils de Mickey qu'il fournisse le lait ? Pourquoi Mickey se charge-t-il d'aller le chercher? De plus, le jeu icono-textuel conçu comme une plaisanterie lexicale et visuelle Mickey, milk; milk cake, Mickey-cake, Mickey way, Milky-way qui ajoute profondeur et espièglerie à la narration, disparaît dans la traduction.

Lorsque Mickey est représenté dans les bouteilles de lait, sa relation avec le lait est montrée de façon visuelle et verbale : Mickey est dans le lait, nageant dans le liquide blanc, le lait est dans son nom, et l'enfant boit le lait (sa bouche est ouverte). La version française simplifie toutes les allusions de l'original : "Que c'est bon et doux le lait ! Vive le lait quand il est frais ! ". Le lien essentiel entre l'enfant et le lait, tout comme la prière éloquente ou la demande de bénédiction sont absents. Le texte cible semble une exaltation hors de propos du lait, superflue dans la structure du texte. Le seul aspect recréé est la sonorité par la rime entre "lait " et " frais ». Cependant, l'aspect expressif est secondaire dans ce passage. 


\section{Le visuel et le verbal résistant à la traduction}

\section{Inter-iconographie}

Nombre de signes iconographiques renvoient à d'autres lieux : bouteilles, sacs, boîtes et cartons dans la cuisine dessinent le profil des gratte-ciels de New York ; les trois boulangers ressemblent à Oliver Hardy(5) ; l'étoile de David est dessinée deux fois sur une boîte de sel (l'artiste est juif) et puis il y a beaucoup d'autres références personnelles(6) ; l'emblème de Mickey sur la page de titre, qui est repris sur la dernière page, rappelle le Mickey Mouse des dessins animés dans les années trente (le nom du personnage est un hommage au héros de Disney) ; enfin, l'apparence du livre In the Night Kitchen évoque Little Nemo in Slumberland(7), la célèbre bande-dessinée de Winsor McCay, qui était publiée dans un quotidien, dont les dessins ont attiré l'attention de Sendak lorsqu'il les a vus dans une exposition au Metropolitan Museum of Art (New-York), en 1965 (Lanes 1980, p.175).

Ces exemples d'inter-iconographie sont profondément ancrés dans le paysage littéraire et culturel américain, ou dans la vie de l'auteur. Ils forment un contexte visuel qui complète la narration mais qui, pour être reconnu et compris, nécessite la connaissance de ce monde visuel. Ces références visuelles risquent de passer inaperçues en raison du processus de traduction car leur étrangeté culturelle ne peut être transposée puisqu'elle ne dépend pas du traducteur. Le niveau inter-iconographique va bien au-delà de l'expérience qu'a l'enfant-lecteur d'un monde étranger et représente une barrière insurmontable pour le traducteur qui ne peut intervenir sur les illustrations. Ainsi, le phénomène des références multiples de l'inter-iconographie ne peut être rendu dans un autre système de signes.

\section{Le verbal dans le visuel}

Le texte dans les images, le "paratexte linguistique " selon la définition de Maria Grazia Margarito, d'après la théorie du paratexte de Gérard Genette (Margarito, 2005), est représenté par les inscriptions sur les objets dans la cuisine et par les onomatopées.

Dans cet album, le paratexte joue un rôle important. Ainsi page 20, les inscriptions sur les boîtes et cartons d'emballage rappellent au lecteur les phases de la croissance d'un enfant (Nières-Chevrel 2003, p. 156) : elles suivent une ligne temporelle de gauche à droite avec les

(5) Selma Lanes explique : " II se trouve que c'est un vieux film de Laurel et Hardy vu à la TV - Nothing but Trouble - qui lui a donné l'idée du trio de boulangers sosies de Hardy dans le livre. " (Lanes 1980, p. 179).

(6) Selma Lanes présente une liste précise des références personnelles de l'artiste à sa vie, comme l'inscription sur un carton de noix de coco "Breveté le 10 juin 1928 " qui est sa date de naissance (Selma 1980, p. 182-183).

(7) Pour en savoir plus sur les ressemblances entre les deux livres, cf. Morpheus. "Meeting McCay." http://springlakemccay.blogspot.com/2007/12/maurice-sendaks-homage.html. Juin 2011. 


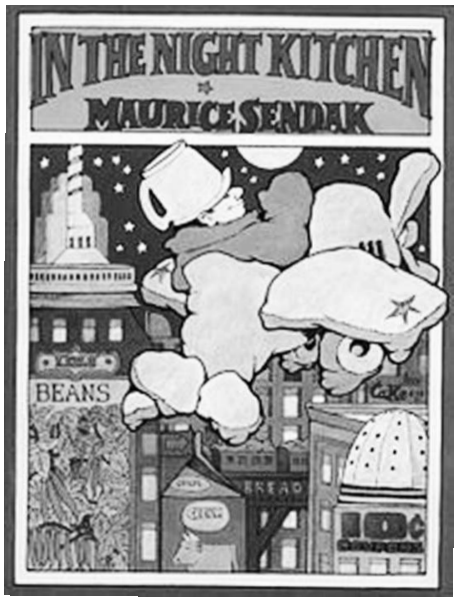

Baby Syrup, Condensed Food, Infant Food, et enfin Rolled White Oats [catégories d'aliments pour enfant selon son âge]. Cette succession symbolise le processus de développement physique et psychologique expérimenté par Mickey dans l'histoire. De plus, page 10, on peut lire, sur le four dans lequel Mickey va être mis à cuire : Mickey oven [Four Mickey]. Cette inscription souligne le lien entre le four et l'enfant : tout comme le lait évoque le liquide amniotique, le four évoque l'environnement in-utero dans lequel Mickey trouve refuge et dont il émerge rempli de force et d'énergie: "I'm not the milk and the milk's not me! I'm Mickey". Ces inscriptions ne sont traduites ni en français ni en italien et leurs messages symboliques demeurent obscurs pour les lecteurs du texte cible. Elles contribuent en outre à créer un cadre étranger qui contredit la stratégie de localisation du texte cible : il est pour le moins curieux de voir Luca, enfant italien qui mange de la "focaccia", ou Mickey, qui apporte du lait pour "le pain brioché " entouré de bouteilles et de boîtes portant des inscriptions en langue étrangère, donc dépourvues de sens. Ces éléments demeurent alors hors du domaine de compréhension, signifiants privés de leur signifiés.

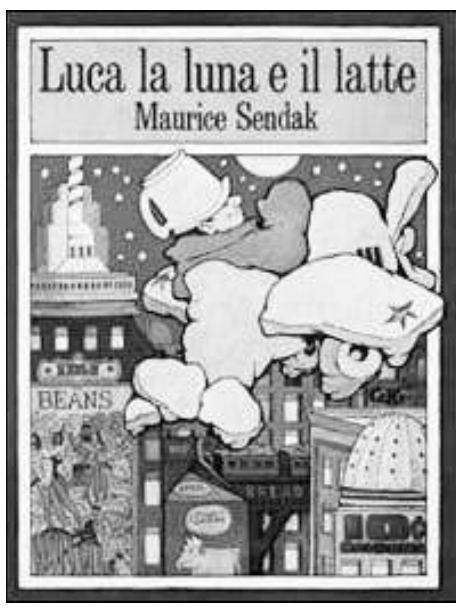

À l'inverse, les onomatopées sont

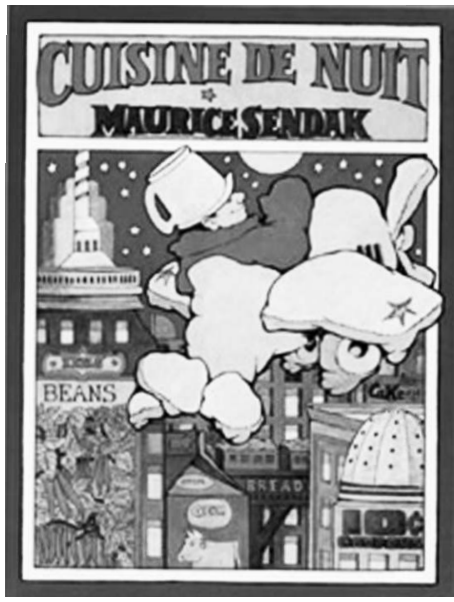
traduites dans les deux langues. Les bruits et les sons ont été rendus en fonction du système sonore de la langue du texte cible. Thump, Dump, Clump, Lump, Bump décrivent les sons qui réveillent Mickey au milieu de la nuit. En italien et en français, ils deviennent des sons sans signification particulière mais visant à donner la même impression déplaisante que ceux du texte source : Scrac, Bum, Trac, Gleng, Deng dans la version italienne ; Roum, Doum, Cloum, Ploum en français. Le Cockadoodledoo du coq est aussi traduit selon le cri conventionnel de l'animal dans les langues cibles et devient donc chicchiricchiiiiii ! en italien et " Cocorico ! " en français. 


\section{Limites techniques}

Les légendes et les bulles imposent une limite à la longueur de la traduction, ce qui complique la tâche des traducteurs lorsque la langue cible tend à foisonner par rapport à la langue d'origine, comme l'italien et le français par rapport à l'anglais. Les traducteurs doivent donc s'arranger pour que leurs textes tiennent dans les espaces prévus.

Le titre italien Luca la luna e il latte étant plus long que l'anglais, on a choisi une autre police pour qu'il entre dans le cadre prévu sur la couverture. Néanmoins, formes et couleurs donnant aux mots une dimension visuelle, le changement de police en modifie l'effet. Les adaptations graphiques du titre le rendent plat et ordinaire tandis que les couleurs, les dimensions et les formes du titre original intriguent et éveillent l'intérêt. De plus, la police du titre est conservée pour toute la traduction, le résultat est donc visuellement moins attractif que celui produit par le texte source.

Le traducteur français, au contraire, préfère supprimer les mots non fonctionnels (dans la) pour conserver la police originale du titre. Ainsi, Cuisine de nuit garde le même aspect que l'original.

\section{Conclusion}

Les traductions italienne et française sont toutes deux décevantes car elles négligent l'interdépendance des dimensions verbale et visuelle qui révèlent les significations sous-jacentes plus complexes de la narration. Sendak décrit la nature psychothérapeutique de l'acte créatif d'In the Night Kitchen en expliquant que "c'est sorti directement du plus profond de mon être et c'était très douloureux de l'en extraire. Oui, vraiment, quelque chose comme la douleur de l'enfantement, et je ne pense pas pouvoir aller plus loin. En fait, c'est surnaturel " (Sendak in Lanes 1980, p. 174). Pourtant, l'intensité des sentiments de Sendak est diluée dans les deux textes cibles. Au final, la traduction a produit un texte radicalement différent de celui proposé par Sendak. Les illustrations sont là comme ornement pour le texte qui perd sa dimension mythique originelle. L'orchestration parfaite entre les mots et les images qui stimule l'imagination de l'enfant et convoque son inconscient, cette exploration du royaume de l'imaginaire ou d'une zone de contact entre signes verbaux et visuels, est rarement recréée dans le texte cible et de ce fait, le génie de Maurice Sendak reste ignoré dans la culture des deux langues cibles.

Traduit de l'anglais par Hélène Ladjadj 


\section{Objet de l'étude}

SENDAK, Maurice. In the Night Kitchen, New York (États-Unis), HarperCollins Publishers, 1970. SENDAK, Maurice. Luca, la luna e il latte, Milan (Italie), Babalibri, 2000. Traduction Selene Maltini. SENDAK, Maurice. Cuisine de nuit, Paris, L'École des loisirs, 1972. Traduction Jean-Henri Potier.

\section{Bibliographie}

AGOSTO, Denise A. "One and Inseparable: Interdependent Storytelling in Picture Storybooks", in Children's Literature in Education 30:4. p. 267-280. 1999.

KRESS, Gunther and van LEEUWEN, Theo. Multimodal Discourse. The Modes and Media of Contemporary Communication. London, Edward Arnold, 2001.

LANES, Selma. The Art of Maurice Sendak. New York (États-Unis), Abradale Press/Harry N. Abrams, Inc. 1980.

MARGARITO, Maria Grazia. "En accompagnement d'images ... d'autres images parfois (notes sur des apartés de la BD) ", Ela 138 (avril-juin 2005), p. 243-255.

MEEK, Margaret. "What Counts as Evidence in Theories of Children's Literature", Peter Hunt (ed.), in Children's Literature: The Development of Criticism. New York (États-Unis), Routledge, 1992. p. 166-182.

MOEBIUS, William. "Introduction to Picturebook Codes". Peter Hunt (ed.), in Children's Literature: The Development of Criticism, New York (États-Unis), Routledge, 1990. p. 131-147. NIĖRES-CHEVREL, Isabelle. "Traduire In the Night Kitchen, ou de la difficile lecture d'un album ", in Meta 48, 2003, p. 1-2, 154-164(8).

OITTINEN, Riitta. Translating for Children. New York (États-Unis), Garland, 2000.

O'SULLIVAN, Emer. "Translating Pictures", in Gillian Lathey (ed.), The Translation of Children's Literature. A Reader. Clevedon, Buffalo, Toronto, Multilingual Matters, 2006. p. 113-121.

SIPE, Lawrence. "How Picture Books Work: A Semiotically Framed Theory of Text-Picture Relationship", in Children's Literature in Education, 29(2), 1998. p. 7-10.

Margherita Ippolito est titulaire d'un Master en Linguistique de corpus appliquée de I'Université de Birmingham (Royaume-Uni) et d'un Doctorat en Traductologie de I'Université de Bari (Italie). Elle enseigne la langue anglaise et la traduction à la Faculté des Langues et littératures étrangères de l'Université de Bari. Ces thèmes de recherche comprennent la traduction de la littérature pour enfants, la traduction de l'interaction texte-image dans les albums illustrés et l'application de la linguistique de corpus à l'analyse des traductions. Elle est l'auteur de l'ouvrage Simplification, Explicitation and Normalization. Corpus-Based Research into English to Italian Translations of Children's Classics (Cambridge Scholars Publishing, Newcastle upon Tyne, 2013).

(8) Cet article est disponible en ligne à l'adresse http://www.erudit.org/revue/meta/2003/v48/n1-2/006964ar.html 